\title{
Jensen and Ostrowski type inequalities for general Lebesgue integral with applications
}

\begin{abstract}
Some inequalities related to Jensen and Ostrowski inequalities for general Lebesgue integral are obtained. Applications for $f$-divergence measure are provided as well.
\end{abstract}

1. Introduction. Let $(\Omega, \mathcal{A}, \mu)$ be a measurable space consisting of a set $\Omega$, a $\sigma$-algebra $\mathcal{A}$ of subsets of $\Omega$ and a countably additive and positive measure $\mu$ on $\mathcal{A}$ with values in $\mathbb{R} \cup\{\infty\}$. Assume, for simplicity, that $\int_{\Omega} d \mu(t)=1$. Consider the Lebesgue space

$$
L(\Omega, \mu):=\left\{f: \Omega \rightarrow \mathbb{R} \mid f \text { is } \mu \text {-measurable and } \int_{\Omega}|f(t)| d \mu(t)<\infty\right\} .
$$

For simplicity of notation we write everywhere in the sequel $\int_{\Omega} w d \mu$ instead of $\int_{\Omega} w(t) d \mu(t)$.

The following reverse of the Jensen's inequality holds [12]:

Theorem 1. Let $\Phi: I \rightarrow \mathbb{R}$ be a continuous convex function on the interval of real numbers $I$ and $m, M \in \mathbb{R}, m<M$ with $[m, M] \subset \stackrel{\circ}{I}$, where $\stackrel{\circ}{I}$ is the interior of I. If $f: \Omega \rightarrow \mathbb{R}$ is $\mu$-measurable, satisfies the bounds

$$
-\infty<m \leq f(t) \leq M<\infty \text { for } \mu \text {-a.e. } t \in \Omega
$$

2010 Mathematics Subject Classification. Primary 26D15; Secondary 94A17.

Key words and phrases. Ostrowski's inequality, Jensen's inequality, f-divergence measures. 
and such that $f, \Phi \circ f \in L(\Omega, \mu)$, then

$$
\begin{aligned}
0 & \leq \int_{\Omega} \Phi \circ f d \mu-\Phi\left(\int_{\Omega} f d \mu\right) \\
& \leq\left(M-\int_{\Omega} f d \mu\right)\left(\int_{\Omega} f d \mu-m\right) \frac{\Phi_{-}^{\prime}(M)-\Phi_{+}^{\prime}(m)}{M-m} \\
& \leq \frac{1}{4}(M-m)\left[\Phi_{-}^{\prime}(M)-\Phi_{+}^{\prime}(m)\right],
\end{aligned}
$$

where $\Phi_{-}^{\prime}$ is the left and $\Phi_{+}^{\prime}$ is the right derivative of the convex function $\Phi$.

For other reverse of Jensen's inequality and applications to divergence measures see [12] and [15].

In 1938, A. Ostrowski [23] proved the following inequality concerning the distance between the integral mean $\frac{1}{b-a} \int_{a}^{b} \Phi(t) d t$ and the value $\Phi(x)$, $x \in[a, b]$.

Theorem 2. Let $\Phi:[a, b] \rightarrow \mathbb{R}$ be continuous on $[a, b]$ and differentiable on $(a, b)$ such that $\Phi^{\prime}:(a, b) \rightarrow \mathbb{R}$ is bounded on $(a, b)$, i.e., $\left\|\Phi^{\prime}\right\|_{\infty}:=$ $\sup _{t \in(a, b)}\left|\Phi^{\prime}(t)\right|<\infty$. Then

$$
\left|\Phi(x)-\frac{1}{b-a} \int_{a}^{b} f(t) d t\right| \leq\left[\frac{1}{4}+\left(\frac{x-\frac{a+b}{2}}{b-a}\right)^{2}\right]\left\|\Phi^{\prime}\right\|_{\infty}(b-a),
$$

for all $x \in[a, b]$ and the constant $\frac{1}{4}$ is the best possible.

For various results related to Ostrowski's inequality see for instance [2], [3], [5]-[18] and the references therein.

Motivated by the above results, in this paper we investigate the magnitude of the quantity

$$
\int_{\Omega} \Phi \circ g d \mu-\Phi(x)-\lambda\left(\int_{\Omega} g d \mu-x\right), x \in[a, b],
$$

for various assumptions on the absolutely continuous function $\Phi$, which in the particular case of $x=\int_{\Omega} g d \mu$ provides some results connected with Jensen's inequality while in the case $\lambda=0$ provides some generalizations of Ostrowski's inequality. Applications for divergence measures are provided as well.

2. Some identities. The following result holds:

Lemma 1. Let $\Phi: I \rightarrow \mathbb{C}$ be an absolutely continuous function on $[a, b] \subset \stackrel{\circ}{\mathrm{I}}$, the interior of $I$. If $g: \Omega \rightarrow[a, b]$ is Lebesgue $\mu$-measurable on $\Omega$ and such 
that $\Phi \circ g, g \in L(\Omega, \mu)$, then we have the equality

$$
\begin{aligned}
& \int_{\Omega} \Phi \circ g d \mu-\Phi(x)-\lambda\left(\int_{\Omega} g d \mu-x\right) \\
& =\int_{\Omega}\left[(g-x) \int_{0}^{1}\left(\Phi^{\prime}((1-s) x+s g)-\lambda\right) d s\right] d \mu
\end{aligned}
$$

for any $\lambda \in \mathbb{C}$ and $x \in[a, b]$.

In particular, we have

$$
\int_{\Omega} \Phi \circ g d \mu-\Phi(x)=\int_{\Omega}\left[(g-x) \int_{0}^{1} \Phi^{\prime}((1-s) x+s g) d s\right] d \mu,
$$

for any $x \in[a, b]$.

Proof. Since $\Phi$ is absolutely continuous on $[a, b]$, then for any $u, v \in[a, b]$ we have

$$
\Phi(u)-\Phi(v)=(u-v) \int_{0}^{1} \Phi^{\prime}((1-s) v+s u) d s .
$$

This implies that

$$
\Phi(g(t))-\Phi(x)=(g(t)-x) \int_{0}^{1} \Phi^{\prime}((1-s) x+s g(t)) d s
$$

for any $t \in \Omega$, or, equivalently

$$
\Phi \circ g-\Phi(x)=(g-x) \int_{0}^{1} \Phi^{\prime}((1-s) x+s g) d s .
$$

Since $\Phi: I \rightarrow \mathbb{C}$ is an absolutely continuous functions on $[a, b]$, the Lebesgue integral over $\mu$ in the right side of (2.1) exists for any $\lambda \in \mathbb{C}$ and $x \in[a, b]$.

Integrating (2.4) over the measure $\mu$ on $\Omega$ and since $\int_{\Omega} d \mu=1$, then we have

$$
\int_{\Omega} \Phi \circ g d \mu-\Phi(x)=\int_{\Omega}\left[(g-x) \int_{0}^{1} \Phi^{\prime}((1-s) x+s g) d s\right] d \mu .
$$

Now, observe that for $\lambda \in \mathbb{C}$ we have

$$
\begin{aligned}
& \int_{\Omega}\left[(g-x) \int_{0}^{1}\left(\Phi^{\prime}((1-s) x+s g)-\lambda\right) d s\right] d \mu \\
& =\int_{\Omega}\left[(g-x)\left(\int_{0}^{1} \Phi^{\prime}((1-s) x+s g) d s-\lambda\right)\right] d \mu \\
& =\int_{\Omega}\left[(g-x) \int_{0}^{1} \Phi^{\prime}((1-s) x+s g) d s\right] d \mu-\lambda \int_{\Omega}(g-x) d \mu \\
& =\int_{\Omega}\left[(g-x) \int_{0}^{1} \Phi^{\prime}((1-s) x+s g) d s\right] d \mu-\lambda\left(\int_{\Omega} g d \mu-x\right) .
\end{aligned}
$$

Making use of (2.5) and (2.6), we deduce the desired result (2.1). 
Remark 1. With the assumptions of Lemma 1 we have

$$
\begin{aligned}
& \int_{\Omega} \Phi \circ g d \mu-\Phi\left(\frac{a+b}{2}\right) \\
& =\int_{\Omega}\left[\left(g-\frac{a+b}{2}\right) \int_{0}^{1} \Phi^{\prime}\left((1-s) \frac{a+b}{2}+s g\right) d s\right] d \mu .
\end{aligned}
$$

Corollary 1. With the assumptions of Lemma 1 we have

$$
\begin{aligned}
& \int_{\Omega} \Phi \circ g d \mu-\Phi\left(\int_{\Omega} g d \mu\right) \\
& =\int_{\Omega}\left[\left(g-\int_{\Omega} g d \mu\right) \int_{0}^{1} \Phi^{\prime}\left((1-s) \int_{\Omega} g d \mu+s g\right) d s\right] d \mu .
\end{aligned}
$$

Proof. We observe that since $g: \Omega \rightarrow[a, b]$ and $\int_{\Omega} d \mu=1$, then $\int_{\Omega} g d \mu \in$ $[a, b]$ and by taking $x=\int_{\Omega} g d \mu$ in $(2.2)$, we get $(2.8)$.

Corollary 2. With the assumptions of Lemma 1 we have

$$
\begin{aligned}
& \int_{\Omega} \Phi \circ g d \mu-\frac{1}{b-a} \int_{a}^{b} \Phi(x) d x-\lambda\left(\int_{\Omega} g d \mu-\frac{a+b}{2}\right) \\
& =\int_{\Omega}\left\{\frac{1}{b-a} \int_{a}^{b}\left[(g-x) \int_{0}^{1}\left(\Phi^{\prime}((1-s) x+s g)-\lambda\right) d s\right] d x\right\} d \mu .
\end{aligned}
$$

Proof. Follows by integrating the identity (2.1) over $x \in[a, b]$, dividing by $b-a>0$ and using Fubini's theorem.

Corollary 3. Let $\Phi: I \rightarrow \mathbb{C}$ be an absolutely continuous function on $[a, b] \subset$ $\stackrel{\circ}{\mathrm{I}}$, the interior of I. If $g, h: \Omega \rightarrow[a, b]$ are Lebesgue $\mu$-measurable on $\Omega$ and such that $\Phi \circ g, \Phi \circ h, g, h \in L(\Omega, \mu)$, then we have the equality

$$
\begin{aligned}
& \int_{\Omega} \Phi \circ g d \mu-\int_{\Omega} \Phi \circ h d \mu-\lambda\left(\int_{\Omega} g d \mu-\int_{\Omega} h d \mu\right) \\
& =\int_{\Omega} \int_{\Omega}\left[(g(t)-h(\tau)) \int_{0}^{1}\left(\Phi^{\prime}((1-s) h(\tau)+s g(t))-\lambda\right) d s\right] \\
& \quad \times d \mu(t) d \mu(\tau)
\end{aligned}
$$

for any $\lambda \in \mathbb{C}$ and $x \in[a, b]$.

In particular, we have

$$
\begin{aligned}
& \int_{\Omega} \Phi \circ g d \mu-\int_{\Omega} \Phi \circ h d \mu \\
& =\int_{\Omega} \int_{\Omega}\left[(g(t)-h(\tau)) \int_{0}^{1} \Phi^{\prime}((1-s) h(\tau)+s g(t)) d s\right] \\
& \quad \times d \mu(t) d \mu(\tau),
\end{aligned}
$$

for any $x \in[a, b]$. 
Proof. From (2.1) we have for any $\tau \in \Omega$ that

$$
\begin{aligned}
& \int_{\Omega} \Phi \circ g d \mu-\Phi(h(\tau))-\lambda\left(\int_{\Omega} g d \mu-\Phi(h(\tau))\right) \\
& =\int_{\Omega}\left[(g-\Phi(h(\tau))) \int_{0}^{1}\left(\Phi^{\prime}((1-s) \Phi(h(\tau))+s g)-\lambda\right) d s\right] d \mu
\end{aligned}
$$

for any $\lambda \in \mathbb{C}$ and $x \in[a, b]$.

Integrating on $\Omega$ over $d \mu(\tau)$ and using Fubini's theorem, we get the desired result (2.10).

Remark 2. The above equality (2.10) can be extended for two measures as follows

$$
\begin{aligned}
& \int_{\Omega_{1}} \Phi \circ g d \mu_{1}-\int_{\Omega_{2}} \Phi \circ h d \mu_{2}-\lambda\left(\int_{\Omega_{1}} g d \mu_{1}-\int_{\Omega_{2}} h d \mu_{2}\right) \\
& =\int_{\Omega_{1}} \int_{\Omega_{2}}\left[(g(t)-h(\tau)) \int_{0}^{1}\left(\Phi^{\prime}((1-s) h(\tau)+s g(t))-\lambda\right) d s\right] \\
& \quad \times d \mu_{1}(t) d \mu_{2}(\tau),
\end{aligned}
$$

for any $\lambda \in \mathbb{C}$ and $x \in[a, b]$ and provided that $\Phi \circ g, g \in L\left(\Omega_{1}, \mu_{1}\right)$ while $\Phi \circ h, h \in L\left(\Omega_{2}, \mu_{2}\right)$.

Remark 3. If $w \geq 0 \mu$-almost everywhere ( $\mu$-a.e.) on $\Omega$ with $\int_{\Omega} w d \mu>0$, then by replacing $d \mu$ with $\frac{w d \mu}{\int_{\Omega} w d \mu}$ in (2.1), we have the weighted equality

$$
\begin{aligned}
& \frac{1}{\int_{\Omega} w d \mu} \int_{\Omega} w(\Phi \circ g) d \mu-\Phi(x)-\lambda\left(\frac{1}{\int_{\Omega} w d \mu} \int_{\Omega} w g d \mu-x\right) \\
& =\frac{1}{\int_{\Omega} w d \mu} \int_{\Omega} w \cdot\left[(g-x) \int_{0}^{1}\left(\Phi^{\prime}((1-s) x+s g)-\lambda\right) d s\right] d \mu
\end{aligned}
$$

for any $\lambda \in \mathbb{C}$ and $x \in[a, b]$, provided $\Phi \circ g, g \in L_{w}(\Omega, \mu)$, where

$$
L_{w}(\Omega, \mu):=\left\{g\left|\int_{\Omega} w\right| g \mid d \mu<\infty\right\}
$$

The other equalities have similar weighted versions. However, the details are omitted.

If we use the discrete measure, then for a function $\Phi: I \rightarrow \mathbb{C}$ which is absolutely continuous on $[a, b] \subset \stackrel{\circ}{I}$, the interior of $I, x_{j} \in[a, b]$ and $p_{j} \geq 0$ with $\sum_{j=1}^{n} p_{j}=1$, we can state the following identity 


$$
\begin{aligned}
& \sum_{j=1}^{n} p_{j} \Phi\left(x_{j}\right)-\Phi(x)-\lambda\left(\sum_{j=1}^{n} p_{j} x_{j}-x\right) \\
& =\sum_{j=1}^{n} p_{j}\left[\left(x_{j}-x\right) \int_{0}^{1}\left(\Phi^{\prime}\left((1-s) x+s x_{j}\right)-\lambda\right) d s\right]
\end{aligned}
$$

for any $\lambda \in \mathbb{C}$ and $x \in[a, b]$.

In particular, we have

$$
\sum_{j=1}^{n} p_{j} \Phi\left(x_{j}\right)-\Phi(x)=\sum_{j=1}^{n} p_{j}\left[\left(x_{j}-x\right) \int_{0}^{1} \Phi^{\prime}\left((1-s) x+s x_{j}\right) d s\right]
$$

for any $x \in[a, b]$ and

$$
\begin{aligned}
& \sum_{j=1}^{n} p_{j} \Phi\left(x_{j}\right)-\Phi\left(\frac{a+b}{2}\right) \\
& =\sum_{j=1}^{n} p_{j}\left[\left(x_{j}-\frac{a+b}{2}\right) \int_{0}^{1} \Phi^{\prime}\left((1-s) \frac{a+b}{2}+s x_{j}\right) d s\right]
\end{aligned}
$$

and

$$
\begin{aligned}
& \sum_{j=1}^{n} p_{j} \Phi\left(x_{j}\right)-\Phi\left(\sum_{k=1}^{n} p_{k} x_{k}\right) \\
& =\sum_{j=1}^{n} p_{j}\left[\left(x_{j}-\sum_{k=1}^{n} p_{k} x_{k}\right) \int_{0}^{1} \Phi^{\prime}\left((1-s) \sum_{k=1}^{n} p_{k} x_{k}+s x_{j}\right) d s\right] .
\end{aligned}
$$

If $x_{j} \in[a, b]$ and $p_{j} \geq 0, j \in\{1, \ldots, n\}$ with $\sum_{j=1}^{n} p_{j}=1$ and if $y_{k} \in[a, b]$ and $q_{k} \geq 0, k \in\{1, \ldots, m\}$ with $\sum_{k=1}^{m} q_{k}=1$, then we can state the following identity as well:

$$
\begin{aligned}
& \sum_{j=1}^{n} p_{j} \Phi\left(x_{j}\right)-\sum_{k=1}^{m} q_{k} \Phi\left(y_{k}\right)-\lambda\left(\sum_{j=1}^{n} p_{j} x_{j}-\sum_{k=1}^{m} q_{k} y_{k}\right) \\
& =\sum_{j=1}^{n} p_{j} \sum_{k=1}^{m} q_{k}\left[\left(x_{j}-y_{k}\right) \int_{0}^{1}\left(\Phi^{\prime}\left((1-s) y_{k}+s x_{j}\right)-\lambda\right) d s\right] .
\end{aligned}
$$

In particular, we have

$$
\begin{aligned}
& \sum_{j=1}^{n} p_{j} \Phi\left(x_{j}\right)-\sum_{k=1}^{m} q_{k} \Phi\left(y_{k}\right) \\
& =\sum_{j=1}^{n} p_{j} \sum_{k=1}^{m} q_{k}\left[\left(x_{j}-y_{k}\right) \int_{0}^{1} \Phi^{\prime}\left((1-s) y_{k}+s x_{j}\right) d s\right] .
\end{aligned}
$$


3. Bounds in terms of $\boldsymbol{p}$-norms. We use the notations

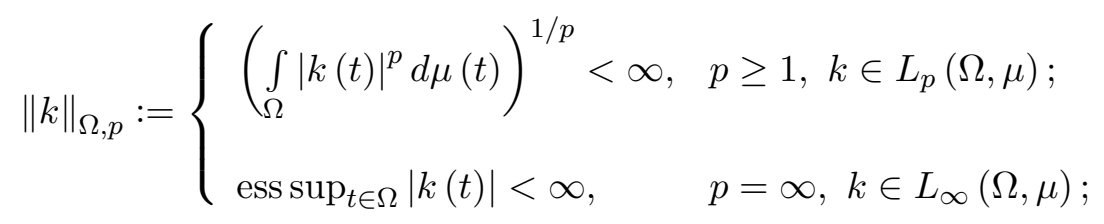

and

$$
\|\Phi\|_{[0,1], p}:= \begin{cases}\left(\int_{0}^{1}|\Phi(s)|^{p} d s\right)^{1 / p}<\infty, & p \geq 1, \Phi \in L_{p}(0,1) \\ {\operatorname{ess} \sup _{s \in[0,1]}|\Phi(s)|<\infty,} \mid p=\infty, \Phi \in L_{\infty}(0,1) .\end{cases}
$$

If we consider the identity function $\ell:[0,1] \rightarrow[0,1], \ell(s)=s$ we have

$$
\int_{0}^{1}\left|\Phi^{\prime}((1-s) x+s g(t))-\lambda\right|^{p} d s=\left\|\Phi^{\prime}((1-\ell) x+\ell g(t))-\lambda\right\|_{[0,1], p}^{p}
$$

and

$$
\underset{s \in[0,1]}{\operatorname{ess} \sup }\left|\Phi^{\prime}((1-s) x+s g(t))-\lambda\right|=\left\|\Phi^{\prime}((1-\ell) x+\ell g(t))-\lambda\right\|_{[0,1], \infty}
$$

for $t \in \Omega$.

Theorem 3. Let $\Phi: I \rightarrow \mathbb{C}$ be an absolutely continuous function on $[a, b] \subset$ $\stackrel{\circ}{I}$, the interior of $I$. If $g: \Omega \rightarrow[a, b]$ is Lebesgue $\mu$-measurable on $\Omega$ and such that $\Phi \circ g, g \in L(\Omega, \mu)$, then

$$
\begin{aligned}
& \left|\int_{\Omega} \Phi \circ g d \mu-\Phi(x)-\lambda\left(\int_{\Omega} g d \mu-x\right)\right| \\
& \quad \leq \int_{\Omega}|g-x|\left\|\Phi^{\prime}((1-\ell) x+\ell g)-\lambda\right\|_{[0,1], 1} d \mu \\
& \quad \leq\left\{\begin{array}{l}
\|g-x\|_{\Omega, \infty}\|\| \Phi^{\prime}((1-\ell) x+\ell g)-\lambda\left\|_{[0,1], 1}\right\|_{\Omega, 1} \\
\|g-x\|_{\Omega, p}\|\| \Phi^{\prime}((1-\ell) x+\ell g)-\lambda\left\|_{[0,1], 1}\right\|_{\Omega, q} \\
p>1, \frac{1}{p}+\frac{1}{q}=1 ; \\
\|g-x\|_{\Omega, 1}\|\| \Phi^{\prime}((1-\ell) x+\ell g)-\lambda\left\|_{[0,1], 1}\right\|_{\Omega, \infty}
\end{array}\right.
\end{aligned}
$$

for any $\lambda \in \mathbb{C}$ and $x \in[a, b]$. In particular, we have 


$$
\begin{aligned}
& \left|\int_{\Omega} \Phi \circ g d \mu-\Phi(x)\right| \\
& \quad \leq \int_{\Omega}|g-x|\left\|\Phi^{\prime}((1-\ell) x+\ell g)\right\|_{[0,1], 1} d \mu \\
& \quad \leq\left\{\begin{array}{l}
\|g-x\|_{\Omega, \infty}\|\| \Phi^{\prime}((1-\ell) x+\ell g)\left\|_{[0,1], 1}\right\|_{\Omega, 1} ; \\
\|g-x\|_{\Omega, p}\|\| \Phi^{\prime}((1-\ell) x+\ell g)\left\|_{[0,1], 1}\right\|_{\Omega, q} \\
p>1, \frac{1}{p}+\frac{1}{q}=1 ; \\
\|g-x\|_{\Omega, 1}\|\| \Phi^{\prime}((1-\ell) x+\ell g)\left\|_{[0,1], 1}\right\|_{\Omega, \infty}
\end{array}\right.
\end{aligned}
$$

for any $x \in[a, b]$.

Proof. Taking the modulus in the equality (2.1), we have

$$
\begin{aligned}
& \left|\int_{\Omega} \Phi \circ g d \mu-\Phi(x)-\lambda\left(\int_{\Omega} g d \mu-x\right)\right| \\
& \quad \leq \int_{\Omega}\left|(g-x) \int_{0}^{1}\left(\Phi^{\prime}((1-s) x+s g)-\lambda\right) d s\right| d \mu \\
& \quad \leq \int_{\Omega}|g-x| \int_{0}^{1}\left|\Phi^{\prime}((1-s) x+s g)-\lambda\right| d s d \mu \\
& \quad=\int_{\Omega}|g-x|\left\|\Phi^{\prime}((1-\ell) x+\ell g)-\lambda\right\|_{[0,1], 1} d \mu
\end{aligned}
$$

for any $\lambda \in \mathbb{C}$ and $x \in[a, b]$.

Utilising Hölder's inequality for the $\mu$-measurable functions $F, G: \Omega \rightarrow \mathbb{C}$,

$$
\left|\int_{\Omega} F G d \mu\right| \leq\left(\int_{\Omega}|F|^{p} d \mu\right)^{1 / p}\left(\int_{\Omega}|G|^{q} d \mu\right)^{1 / q}, p>1, \frac{1}{p}+\frac{1}{q}=1
$$

and

$$
\left|\int_{\Omega} F G d \mu\right| \leq \operatorname{essup}_{t \in \Omega}|F(t)| \int_{\Omega}|G| d \mu
$$

we get from (3.3) the desired result (3.1).

Remark 4. If we take $x=\frac{a+b}{2}$ in (3.1), then we get

$$
\begin{aligned}
& \left|\int_{\Omega} \Phi \circ g d \mu-\Phi\left(\frac{a+b}{2}\right)-\lambda\left(\int_{\Omega} g d \mu-\frac{a+b}{2}\right)\right| \\
& \quad \leq \int_{\Omega}\left|g-\frac{a+b}{2}\right|\left\|\Phi^{\prime}\left((1-\ell) \frac{a+b}{2}+\ell g\right)-\lambda\right\|_{[0,1], 1} d \mu
\end{aligned}
$$




$$
\leq\left\{\begin{array}{l}
\left\|g-\frac{a+b}{2}\right\|_{\Omega, \infty}\|\| \Phi^{\prime}\left((1-\ell) \frac{a+b}{2}+\ell g\right)-\lambda\left\|_{[0,1], 1}\right\|_{\Omega, 1} ; \\
\left\|g-\frac{a+b}{2}\right\|_{\Omega, p}\|\| \Phi^{\prime}\left((1-\ell) \frac{a+b}{2}+\ell g\right)-\lambda\left\|_{[0,1], 1}\right\|_{\Omega, q} \\
p>1, \frac{1}{p}+\frac{1}{q}=1 ; \\
\left\|g-\frac{a+b}{2}\right\|_{\Omega, 1}\|\| \Phi^{\prime}\left((1-\ell) \frac{a+b}{2}+\ell g\right)-\lambda\left\|_{[0,1], 1}\right\|_{\Omega, \infty} ;
\end{array}\right.
$$

for any $\lambda \in \mathbb{C}$ and, in particular, for $\lambda=0$ we have

$$
\begin{aligned}
& \left|\int_{\Omega} \Phi \circ g d \mu-\Phi\left(\frac{a+b}{2}\right)\right| \\
& \quad \leq \int_{\Omega}\left|g-\frac{a+b}{2}\right|\left\|\Phi^{\prime}\left((1-\ell) \frac{a+b}{2}+\ell g\right)\right\|_{[0,1], 1} d \mu \\
& \quad \leq\left\{\begin{array}{l}
\left\|g-\frac{a+b}{2}\right\|_{\Omega, \infty}\|\| \Phi^{\prime}\left((1-\ell) \frac{a+b}{2}+\ell g\right)\left\|_{[0,1], 1}\right\|_{\Omega, 1} \\
\left\|g-\frac{a+b}{2}\right\|_{\Omega, p}\|\| \Phi^{\prime}\left((1-\ell) \frac{a+b}{2}+\ell g\right)\left\|_{[0,1], 1}\right\|_{\Omega, q} \\
p>1, \frac{1}{p}+\frac{1}{q}=1 ; \\
\left\|g-\frac{a+b}{2}\right\|_{\Omega, 1}\|\| \Phi^{\prime}\left((1-\ell) \frac{a+b}{2}+\ell g\right)\left\|_{[0,1], 1}\right\|_{\Omega, \infty}
\end{array}\right.
\end{aligned}
$$

If we take $x=\int_{\Omega} g d \mu$ in (3.1), then we get

$$
\begin{aligned}
& \left|\int_{\Omega} \Phi \circ g d \mu-\Phi\left(\int_{\Omega} g d \mu\right)\right| \\
& \leq \int_{\Omega}\left|g-\int_{\Omega} g d \mu\right|\left\|\Phi^{\prime}\left((1-\ell) \int_{\Omega} g d \mu+\ell g\right)-\lambda\right\|_{[0,1], 1} d \mu \\
& \leq\left\{\begin{array}{l}
\left\|g-\int_{\Omega} g d \mu\right\|_{\Omega, \infty}\|\| \Phi^{\prime}\left((1-\ell) \int_{\Omega} g d \mu+\ell g\right)-\lambda\left\|_{[0,1], 1}\right\|_{\Omega, 1} ; \\
\left\|g-\int_{\Omega} g d \mu\right\|_{\Omega, p}\|\| \Phi^{\prime}\left((1-\ell) \int_{\Omega} g d \mu+\ell g\right)-\lambda\left\|_{[0,1], 1}\right\|_{\Omega, q} \\
p>1, \frac{1}{p}+\frac{1}{q}=1 ; \\
\left\|g-\int_{\Omega} g d \mu\right\|_{\Omega, 1}\|\| \Phi^{\prime}\left((1-\ell) \int_{\Omega} g d \mu+\ell g\right)-\lambda\left\|_{[0,1], 1}\right\|_{\Omega, \infty} ;
\end{array}\right.
\end{aligned}
$$

for any $\lambda \in \mathbb{C}$ and, in particular, for $\lambda=0$ we have 


$$
\begin{aligned}
& \left|\int_{\Omega} \Phi \circ g d \mu-\Phi\left(\int_{\Omega} g d \mu\right)\right| \\
& \quad \leq \int_{\Omega}\left|g-\int_{\Omega} g d \mu\right|\left\|\Phi^{\prime}\left((1-\ell) \int_{\Omega} g d \mu+\ell g\right)\right\|_{[0,1], 1} d \mu \\
& \quad \leq\left\{\begin{array}{l}
\left\|g-\int_{\Omega} g d \mu\right\|_{\Omega, \infty}\|\| \Phi^{\prime}\left((1-\ell) \int_{\Omega} g d \mu+\ell g\right)\left\|_{[0,1], 1}\right\|_{\Omega, 1} ; \\
\left\|g-\int_{\Omega} g d \mu\right\|_{\Omega, p}\|\| \Phi^{\prime}\left((1-\ell) \int_{\Omega} g d \mu+\ell g\right)\left\|_{[0,1], 1}\right\|_{\Omega, q} \\
\left\|g-\int_{\Omega} g d \mu\right\|_{\Omega, 1}\|\| \Phi^{\prime}\left((1-\ell) \int_{\Omega} g d \mu+\ell g\right)\left\|_{[0,1], 1}\right\|_{\Omega, \infty}
\end{array}\right.
\end{aligned}
$$

Corollary 4. Let $\Phi: I \rightarrow \mathbb{C}$ be an absolutely continuous function on $[a, b] \subset$ $I$, the interior of $I$. If $g: \Omega \rightarrow[a, b]$ is Lebesgue $\mu$-measurable on $\Omega$ and such that $\Phi \circ g, g \in L(\Omega, \mu)$, then

$$
\left|\int_{\Omega} \Phi \circ g d \mu-\Phi(x)\right| \leq\left\|\Phi^{\prime}\right\|_{[a, b], \infty} \int_{\Omega}|g-x| d \mu
$$

for any $x \in[a, b]$.

In particular, we have

$$
\left|\int_{\Omega} \Phi \circ g d \mu-\Phi\left(\frac{a+b}{2}\right)\right| \leq\left\|\Phi^{\prime}\right\|_{[a, b], \infty} \int_{\Omega}\left|g-\frac{a+b}{2}\right| d \mu
$$

and

$$
\left|\int_{\Omega} \Phi \circ g d \mu-\Phi\left(\int_{\Omega} g d \mu\right)\right| \leq\left\|\Phi^{\prime}\right\|_{[a, b], \infty} \int_{\Omega}\left|g-\int_{\Omega} g d \mu\right| d \mu .
$$

Proof. We have from (3.1) that

$$
\left|\int_{\Omega} \Phi \circ g d \mu-\Phi(x)\right| \leq \int_{\Omega}|g-x|\left(\int_{0}^{1}\left|\Phi^{\prime}((1-s) x+s g)\right| d s\right) d \mu
$$

for any $x \in[a, b]$.

However, for any $t \in \Omega$ and almost every $s \in[0,1]$ we have

$$
\left|\Phi^{\prime}((1-s) x+s g(t))\right| \leq \underset{u \in[a, b]}{\operatorname{ess} \sup }\left|\Phi^{\prime}(u)\right|=\left\|\Phi^{\prime}\right\|_{[a, b], \infty},
$$

for any $x \in[a, b]$.

Making use of (3.11), we get (3.8).

Remark 5. We remark that the quantity from Corollary 4

$$
\delta_{\mu}(g, x):=\int_{\Omega}|g-x| d \mu
$$

cannot be computed in general. 
However, in the case when $\Omega=[a, b], g:[a, b] \rightarrow[a, b], g(t)=t$ and $\mu(t)=\frac{1}{b-a} d t$, we have

$$
\begin{aligned}
\delta_{\mu}(g, x) & :=\frac{1}{b-a} \int_{a}^{b}|t-x| d t=\frac{1}{b-a}\left[\int_{a}^{x}(x-t) d t+\int_{x}^{b}(t-x) d t\right] \\
& =\frac{1}{b-a}\left[(x-a)^{2}+(b-x)^{2}\right] \\
& =\left[\frac{1}{4}+\left(\frac{x-\frac{a+b}{2}}{b-a}\right)^{2}\right](b-a),
\end{aligned}
$$

where $x \in[a, b]$.

Utilising the inequality (3.8), we get Ostrowski's inequality

$$
\begin{aligned}
& \left|\frac{1}{b-a} \int_{a}^{b} \Phi(t) d t-\Phi(x)\right| \\
& \quad \leq\left\|\Phi^{\prime}\right\|_{[a, b], \infty}\left[\frac{1}{4}+\left(\frac{x-\frac{a+b}{2}}{b-a}\right)^{2}\right](b-a)
\end{aligned}
$$

for any $x \in[a, b]$.

From the inequalities (3.9) and (3.10) we get the midpoint inequality

$$
\left|\frac{1}{b-a} \int_{a}^{b} \Phi(t) d t-\Phi\left(\frac{a+b}{2}\right)\right| \leq \frac{1}{4}\left\|\Phi^{\prime}\right\|_{[a, b], \infty}(b-a) .
$$

Remark 6. If we consider the dispersion or the standard variation

$$
\sigma_{\mu}(g):=\left(\int_{\Omega}\left(g-\int_{\Omega} g d \mu\right)^{2} d \mu\right)^{1 / 2}=\left(\int_{\Omega} g^{2} d \mu-\left(\int_{\Omega} g d \mu\right)^{2}\right)^{1 / 2}
$$

then by (3.10) we have the inequalities

$$
\begin{aligned}
\left|\int_{\Omega} \Phi \circ g d \mu-\Phi\left(\int_{\Omega} g d \mu\right)\right| & \leq\left\|\Phi^{\prime}\right\|_{[a, b], \infty} \delta_{\mu}\left(g, \int_{\Omega} g d \mu\right) \\
& \leq\left\|\Phi^{\prime}\right\|_{[a, b], \infty} \sigma_{\mu}(g) .
\end{aligned}
$$

In general, we have by Cauchy-Bunyakovsky-Schwarz's inequality that

$$
\delta_{\mu}(g, x):=\int_{\Omega}|g-x| d \mu \leq\left(\int_{\Omega}(g-x)^{2} d \mu\right)^{1 / 2} .
$$

Since

$$
\begin{aligned}
& \int_{\Omega}(g-x)^{2} d \mu=\int_{\Omega}\left(g-\int_{\Omega} g d \mu+\int_{\Omega} g d \mu-x\right)^{2} d \mu \\
& =\int_{\Omega}\left(g-\int_{\Omega} g d \mu\right)^{2} d \mu+2 \int_{\Omega}\left(g-\int_{\Omega} g d \mu\right)\left(\int_{\Omega} g d \mu-x\right) d \mu
\end{aligned}
$$




$$
\begin{aligned}
& +\int_{\Omega}\left(\int_{\Omega} g d \mu-x\right)^{2} d \mu \\
& =\int_{\Omega}\left(g-\int_{\Omega} g d \mu\right)^{2} d \mu+\left(\int_{\Omega} g d \mu-x\right)^{2}
\end{aligned}
$$

for any $x \in[a, b]$, then by (3.8) and (3.15) we get the inequalities

$$
\begin{aligned}
\left|\int_{\Omega} \Phi \circ g d \mu-\Phi(x)\right| & \leq\left\|\Phi^{\prime}\right\|_{[a, b], \infty} \delta_{\mu}(g, x) \\
& \leq\left\|\Phi^{\prime}\right\|_{[a, b], \infty}\left[\sigma_{\mu}^{2}(g)+\left(\int_{\Omega} g d \mu-x\right)^{2}\right]^{1 / 2}
\end{aligned}
$$

for any $x \in[a, b]$.

If we use the discrete measure, then from (3.16) we have

$$
\begin{aligned}
& \left|\sum_{j=1}^{n} p_{j} \Phi\left(x_{j}\right)-\Phi(x)\right| \\
& \leq\left\|\Phi^{\prime}\right\|_{[a, b], \infty} \sum_{j=1}^{n} p_{j}\left|x_{j}-x\right| \\
& \leq\left\|\Phi^{\prime}\right\|_{[a, b], \infty}\left[\sum_{j=1}^{n} p_{j} x_{j}^{2}-\left(\sum_{j=1}^{n} p_{j} x_{j}\right)^{2}+\left(\sum_{j=1}^{n} p_{j} x_{j}-x\right)^{2}\right]^{1 / 2},
\end{aligned}
$$

for any $x \in[a, b]$, where $x_{j} \in[a, b]$ and $p_{j} \geq 0$ with $\sum_{j=1}^{n} p_{j}=1$.

In particular, we have

$$
\begin{aligned}
\left|\sum_{j=1}^{n} p_{j} \Phi\left(x_{j}\right)-\Phi\left(\frac{a+b}{2}\right)\right| & \leq\left\|\Phi^{\prime}\right\|_{[a, b], \infty} \sum_{j=1}^{n} p_{j}\left|x_{j}-\frac{a+b}{2}\right| \\
& \leq \frac{1}{2}(b-a)\left\|\Phi^{\prime}\right\|_{[a, b], \infty}
\end{aligned}
$$

and

$$
\begin{aligned}
& \left|\sum_{j=1}^{n} p_{j} \Phi\left(x_{j}\right)-\Phi\left(\sum_{k=1}^{n} p_{k} x_{k}\right)\right| \leq\left\|\Phi^{\prime}\right\|_{[a, b], \infty} \sum_{j=1}^{n} p_{j}\left|x_{j}-\sum_{k=1}^{n} p_{k} x_{k}\right| \\
& \leq\left\|\Phi^{\prime}\right\|_{[a, b], \infty}\left[\sum_{j=1}^{n} p_{j} x_{j}^{2}-\left(\sum_{j=1}^{n} p_{j} x_{j}\right)^{2}\right]^{1 / 2} \\
& \leq \frac{1}{2}(b-a)\left\|\Phi^{\prime}\right\|_{[a, b], \infty} .
\end{aligned}
$$


4. Inequalities for bounded derivatives. Now, for $\gamma, \Gamma \in \mathbb{C}$ and $[a, b]$ an interval of real numbers, define the sets of complex-valued functions

$$
\begin{aligned}
& \bar{U}_{[a, b]}(\gamma, \Gamma) \\
& :=\{f:[a, b] \rightarrow \mathbb{C} \mid \operatorname{Re}[(\Gamma-f(t))(\overline{f(t)}-\bar{\gamma})] \geq 0 \text { for a.e. } t \in[a, b]\}
\end{aligned}
$$

and

$$
\begin{aligned}
& \bar{\Delta}_{[a, b]}(\gamma, \Gamma) \\
& :=\left\{f:[a, b] \rightarrow \mathbb{C}|| f(t)-\frac{\gamma+\Gamma}{2}\left|\leq \frac{1}{2}\right| \Gamma-\gamma \mid \text { for a.e. } t \in[a, b]\right\} .
\end{aligned}
$$

The following representation result may be stated.

Proposition 1. For any $\gamma, \Gamma \in \mathbb{C}, \gamma \neq \Gamma$, we have that $\bar{U}_{[a, b]}(\gamma, \Gamma)$ and $\bar{\Delta}_{[a, b]}(\gamma, \Gamma)$ are nonempty, convex and closed sets and

$$
\bar{U}_{[a, b]}(\gamma, \Gamma)=\bar{\Delta}_{[a, b]}(\gamma, \Gamma) .
$$

Proof. We observe that for any $z \in \mathbb{C}$ we have the equivalence

$$
\left|z-\frac{\gamma+\Gamma}{2}\right| \leq \frac{1}{2}|\Gamma-\gamma|
$$

if and only if

$$
\operatorname{Re}[(\Gamma-z)(\bar{z}-\bar{\gamma})] \geq 0 .
$$

This follows by the equality

$$
\frac{1}{4}|\Gamma-\gamma|^{2}-\left|z-\frac{\gamma+\Gamma}{2}\right|^{2}=\operatorname{Re}[(\Gamma-z)(\bar{z}-\bar{\gamma})]
$$

that holds for any $z \in \mathbb{C}$.

The equality (3.1) is thus a simple consequence of this fact.

On making use of the complex numbers field properties, we can also state that:

Corollary 5. For any $\gamma, \Gamma \in \mathbb{C}, \gamma \neq \Gamma$, we have

$$
\begin{aligned}
& \bar{U}_{[a, b]}(\gamma, \Gamma) \\
& =\{f:[a, b] \rightarrow \mathbb{C} \mid(\operatorname{Re} \Gamma-\operatorname{Re} f(t))(\operatorname{Re} f(t)-\operatorname{Re} \gamma) \\
& \quad+(\operatorname{Im} \Gamma-\operatorname{Im} f(t))(\operatorname{Im} f(t)-\operatorname{Im} \gamma) \geq 0 \text { for a.e. } t \in[a, b]\} .
\end{aligned}
$$

Now, if we assume that $\operatorname{Re}(\Gamma) \geq \operatorname{Re}(\gamma)$ and $\operatorname{Im}(\Gamma) \geq \operatorname{Im}(\gamma)$, then we can define the following set of functions as well:

$$
\begin{array}{r}
\bar{S}_{[a, b]}(\gamma, \Gamma):=\{f:[a, b] \rightarrow \mathbb{C} \mid \operatorname{Re}(\Gamma) \geq \operatorname{Re} f(t) \geq \operatorname{Re}(\gamma) \\
\text { and } \operatorname{Im}(\Gamma) \geq \operatorname{Im} f(t) \geq \operatorname{Im}(\gamma) \text { for a.e. } t \in[a, b]\} .
\end{array}
$$

One can easily observe that $\bar{S}_{[a, b]}(\gamma, \Gamma)$ is closed, convex and

$$
\emptyset \neq \bar{S}_{[a, b]}(\gamma, \Gamma) \subseteq \bar{U}_{[a, b]}(\gamma, \Gamma) .
$$


The following result holds:

Theorem 4. Let $\Phi: I \rightarrow \mathbb{C}$ be an absolutely continuous function on $[a, b] \subset \stackrel{\circ}{I}$, the interior of $I$. For some $\gamma, \Gamma \in \mathbb{C}, \gamma \neq \Gamma$, assume that $\Phi^{\prime} \in \bar{U}_{[a, b]}(\gamma, \Gamma)=\bar{\Delta}_{[a, b]}(\gamma, \Gamma)$. If $g: \Omega \rightarrow[a, b]$ is Lebesgue $\mu$-measurable on $\Omega$ and such that $\Phi \circ g, g \in L(\Omega, \mu)$, then we have the inequality

$$
\begin{aligned}
& \left|\int_{\Omega} \Phi \circ g d \mu-\Phi(x)-\frac{\gamma+\Gamma}{2}\left(\int_{\Omega} g d \mu-x\right)\right| \\
& \leq \frac{1}{2}|\Gamma-\gamma| \int_{\Omega}|g-x| d \mu
\end{aligned}
$$

for any $x \in[a, b]$.

In particular, we have

$$
\begin{aligned}
& \left|\int_{\Omega} \Phi \circ g d \mu-\Phi\left(\frac{a+b}{2}\right)-\frac{\gamma+\Gamma}{2}\left(\int_{\Omega} g d \mu-\frac{a+b}{2}\right)\right| \\
& \leq \frac{1}{2}|\Gamma-\gamma| \int_{\Omega}\left|g-\frac{a+b}{2}\right| d \mu \leq \frac{1}{4}(b-a)|\Gamma-\gamma|
\end{aligned}
$$

and

$$
\begin{aligned}
\left|\int_{\Omega} \Phi \circ g d \mu-\Phi\left(\int_{\Omega} g d \mu\right)\right| & \leq \frac{1}{2}|\Gamma-\gamma| \int_{\Omega}\left|g-\int_{\Omega} g d \mu\right| d \mu \\
& \leq \frac{1}{2}|\Gamma-\gamma|\left(\int_{\Omega} g^{2} d \mu-\left(\int_{\Omega} g d \mu\right)^{2}\right)^{1 / 2} \\
& \leq \frac{1}{4}(b-a)|\Gamma-\gamma| .
\end{aligned}
$$

Proof. By the equality (2.1) for $\lambda=\frac{\gamma+\Gamma}{2}$ we have

$$
\begin{aligned}
& \int_{\Omega} \Phi \circ g d \mu-\Phi(x)-\frac{\gamma+\Gamma}{2}\left(\int_{\Omega} g d \mu-x\right) \\
& =\int_{\Omega}\left[(g-x) \int_{0}^{1}\left(\Phi^{\prime}((1-s) x+s g)-\frac{\gamma+\Gamma}{2}\right) d s\right] d \mu .
\end{aligned}
$$

Since $\Phi^{\prime} \in \bar{\Delta}_{[a, b]}(\gamma, \Gamma)$, we have

$$
\left|\Phi^{\prime}((1-s) x+s g(t))-\frac{\gamma+\Gamma}{2}\right| \leq \frac{1}{2}|\Gamma-\gamma|
$$

for a.e. $s \in[0,1]$ and for any $x \in[a, b]$ and any $t \in \Omega$.

Integrating (4.9) over $s$ on $[0,1]$, we get

$$
\int_{0}^{1}\left|\Phi^{\prime}((1-s) x+s g(t))-\frac{\gamma+\Gamma}{2}\right| d s \leq \frac{1}{2}|\Gamma-\gamma|
$$

for any $x \in[a, b]$ and any $t \in \Omega$. 
Taking the modulus in (4.8), we get via (4.10) that

$$
\begin{aligned}
& \left|\int_{\Omega} \Phi \circ g d \mu-\Phi(x)-\frac{\gamma+\Gamma}{2}\left(\int_{\Omega} g d \mu-x\right)\right| \\
& \leq \int_{\Omega}\left[|g-x|\left|\int_{0}^{1}\left(\Phi^{\prime}((1-s) x+s g)-\frac{\gamma+\Gamma}{2}\right) d s\right|\right] d \mu \\
& \leq \int_{\Omega}\left[|g-x| \int_{0}^{1}\left|\Phi^{\prime}((1-s) x+s g(t))-\frac{\gamma+\Gamma}{2}\right| d s\right] d \mu \\
& \leq \frac{1}{2}|\Gamma-\gamma| \int_{\Omega}|g-x| d \mu
\end{aligned}
$$

and the proof of (4.5) is completed.

Corollary 6. Let $\Phi: I \rightarrow \mathbb{R}$ be a convex function on $[a, b] \subset \stackrel{\circ}{I}$, the interior of I. If $g: \Omega \rightarrow[a, b]$ is Lebesgue $\mu$-measurable on $\Omega$ and such that $\Phi \circ g$, $g \in L(\Omega, \mu)$, then we have the inequality

$$
\begin{aligned}
& \left|\int_{\Omega} \Phi \circ g d \mu-\Phi(x)-\frac{\Phi_{+}^{\prime}(a)+\Phi_{-}^{\prime}(b)}{2}\left(\int_{\Omega} g d \mu-x\right)\right| \\
& \leq \frac{1}{2}\left[\Phi_{-}^{\prime}(b)-\Phi_{+}^{\prime}(a)\right] \int_{\Omega}|g-x| d \mu
\end{aligned}
$$

for any $x \in[a, b]$.

In particular, we have

$$
\begin{aligned}
& \left|\int_{\Omega} \Phi \circ g d \mu-\Phi\left(\frac{a+b}{2}\right)-\frac{\Phi_{+}^{\prime}(a)+\Phi_{-}^{\prime}(b)}{2}\left(\int_{\Omega} g d \mu-\frac{a+b}{2}\right)\right| \\
& \leq \frac{1}{2}\left[\Phi_{-}^{\prime}(b)-\Phi_{+}^{\prime}(a)\right] \int_{\Omega}\left|g-\frac{a+b}{2}\right| d \mu \\
& \leq \frac{1}{4}(b-a)\left[\Phi_{-}^{\prime}(b)-\Phi_{+}^{\prime}(a)\right]
\end{aligned}
$$

and

$$
\begin{aligned}
0 \leq \int_{\Omega} \Phi \circ g d \mu & -\Phi\left(\int_{\Omega} g d \mu\right) \\
& \leq \frac{1}{2}\left[\Phi_{-}^{\prime}(b)-\Phi_{+}^{\prime}(a)\right] \int_{\Omega}\left|g-\int_{\Omega} g d \mu\right| d \mu .
\end{aligned}
$$

The discrete case is as follows:

Remark 7. Let $\Phi: I \rightarrow \mathbb{C}$ be an absolutely continuous function on $[a, b] \subset$ $\stackrel{\circ}{I}$, the interior of $I$. For some $\gamma, \Gamma \in \mathbb{C}, \gamma \neq \Gamma$, assume that $\Phi^{\prime} \in \bar{\Delta}_{[a, b]}(\gamma, \Gamma)$. 
If $x_{j} \in[a, b]$ and $p_{j} \geq 0$ with $\sum_{j=1}^{n} p_{j}=1$ then we have the inequality

$$
\begin{aligned}
& \left|\sum_{j=1}^{n} p_{j} \Phi\left(x_{j}\right)-\Phi(x)-\frac{\gamma+\Gamma}{2}\left(\sum_{k=1}^{n} p_{k} x_{k}-x\right)\right| \\
& \quad \leq \frac{1}{2}|\Gamma-\gamma| \sum_{j=1}^{n} p_{j}\left|x_{j}-x\right|
\end{aligned}
$$

for any $x \in[a, b]$.

In particular, we have

$$
\begin{gathered}
\left|\sum_{j=1}^{n} p_{j} \Phi\left(x_{j}\right)-\Phi\left(\frac{a+b}{2}\right)-\frac{\gamma+\Gamma}{2}\left(\sum_{k=1}^{n} p_{k} x_{k}-\frac{a+b}{2}\right)\right| \\
\leq \frac{1}{2}|\Gamma-\gamma| \sum_{j=1}^{n} p_{j}\left|x_{j}-\frac{a+b}{2}\right| \leq \frac{1}{4}(b-a)|\Gamma-\gamma|
\end{gathered}
$$

and

$$
\begin{aligned}
& \left|\sum_{j=1}^{n} p_{j} \Phi\left(x_{j}\right)-\Phi\left(\sum_{k=1}^{n} p_{k} x_{k}\right)\right| \leq \frac{1}{2}|\Gamma-\gamma| \sum_{j=1}^{n} p_{j}\left|x_{j}-\sum_{k=1}^{n} p_{k} x_{k}\right| \\
& \leq \frac{1}{2}|\Gamma-\gamma|\left(\sum_{j=1}^{n} p_{j} x_{j}^{2}-\left(\sum_{k=1}^{n} p_{k} x_{k}\right)^{2}\right)^{1 / 2} \leq \frac{1}{4}(b-a)|\Gamma-\gamma| .
\end{aligned}
$$

If $\Phi: I \rightarrow \mathbb{R}$ is a convex function on $[a, b]$, then we have

$$
\begin{aligned}
& \left|\sum_{j=1}^{n} p_{j} \Phi\left(x_{j}\right)-\Phi(x)-\frac{\Phi_{+}^{\prime}(a)+\Phi_{-}^{\prime}(b)}{2}\left(\sum_{k=1}^{n} p_{k} x_{k}-x\right)\right| \\
& \leq \frac{1}{2}\left[\Phi_{-}^{\prime}(b)-\Phi_{+}^{\prime}(a)\right] \sum_{j=1}^{n} p_{j}\left|x_{j}-x\right|
\end{aligned}
$$

for any $x \in[a, b]$.

In particular, we have

$$
\begin{aligned}
& \left|\sum_{j=1}^{n} p_{j} \Phi\left(x_{j}\right)-\Phi\left(\frac{a+b}{2}\right)-\frac{\Phi_{+}^{\prime}(a)+\Phi_{-}^{\prime}(b)}{2}\left(\sum_{k=1}^{n} p_{k} x_{k}-\frac{a+b}{2}\right)\right| \\
& \leq \frac{1}{2}\left[\Phi_{-}^{\prime}(b)-\Phi_{+}^{\prime}(a)\right] \sum_{j=1}^{n} p_{j}\left|x_{j}-\frac{a+b}{2}\right| \\
& \leq \frac{1}{4}(b-a)\left[\Phi_{-}^{\prime}(b)-\Phi_{+}^{\prime}(a)\right] .
\end{aligned}
$$


5. Applications for $\boldsymbol{f}$-divergence. Assume that a set $\Omega$ and the $\sigma$-finite measure $\mu$ are given. Consider the set of all probability densities on $\mu$ to be $\mathcal{P}:=\left\{p \mid p: \Omega \rightarrow \mathbb{R}, p(t) \geq 0, \int_{\Omega} p(t) d \mu(t)=1\right\}$. The Kullback-Leibler divergence [22] is well known among the information divergences. It is defined as:

$$
D_{K L}(p, q):=\int_{\Omega} p(t) \ln \left[\frac{p(t)}{q(t)}\right] d \mu(t), \quad p, q \in \mathcal{P},
$$

where $\ln$ is to base $e$.

In Information Theory and Statistics, various divergences are applied in addition to the Kullback-Leibler divergence. These are the: variation distance $D_{v}$, Hellinger distance $D_{H}[19], \chi^{2}$-divergence $D_{\chi^{2}}, \alpha$-divergence $D_{\alpha}$, Bhattacharyya distance $D_{B}[1]$, harmonic distance $D_{H a}$, Jeffrey's distance $D_{J}[20]$, triangular discrimination $D_{\Delta}[25]$, etc. They are defined as follows:

$$
D_{v}(p, q):=\int_{\Omega}|p(t)-q(t)| d \mu(t), \quad p, q \in \mathcal{P} ;
$$

$$
\begin{gathered}
D_{H}(p, q):=\int_{\Omega}|\sqrt{p(t)}-\sqrt{q(t)}| d \mu(t), \quad p, q \in \mathcal{P} ; \\
D_{\chi^{2}}(p, q):=\int_{\Omega} p(t)\left[\left(\frac{q(t)}{p(t)}\right)^{2}-1\right] d \mu(t), \quad p, q \in \mathcal{P} ; \\
D_{\alpha}(p, q):=\frac{4}{1-\alpha^{2}}\left[1-\int_{\Omega}[p(t)]^{\frac{1-\alpha}{2}}[q(t)]^{\frac{1+\alpha}{2}} d \mu(t)\right], p, q \in \mathcal{P} ; \\
D_{B}(p, q):=\int_{\Omega} \sqrt{p(t) q(t)} d \mu(t), p, q \in \mathcal{P} ; \\
D_{H a}(p, q):=\int_{\Omega} \frac{2 p(t) q(t)}{p(t)+q(t)} d \mu(t), \quad p, q \in \mathcal{P} ; \\
D_{J}(p, q):=\int_{\Omega}[p(t)-q(t)] \ln \left[\frac{p(t)}{q(t)}\right] d \mu(t), p, q \in \mathcal{P} ; \\
D_{\Delta}(p, q):=\int_{\Omega} \frac{[p(t)-q(t)]^{2}}{p(t)+q(t)} d \mu(t), \quad p, q \in \mathcal{P} .
\end{gathered}
$$

For other divergence measures, see the paper [21] by Kapur or the book online [24] by Taneja.

Csiszár $f$-divergence is defined as follows [4]

$$
I_{f}(p, q):=\int_{\Omega} p(t) f\left[\frac{q(t)}{p(t)}\right] d \mu(t), p, q \in \mathcal{P},
$$


where $f$ is convex on $(0, \infty)$. It is assumed that $f(u)$ is zero and strictly convex at $u=1$. By appropriately defining this convex function, various divergences are derived. Most of the above distances (5.1)-(5.9) are particular instances of Csiszár $f$-divergence. There are also many others which are not in this class (see for example [24]).

The following result holds:

Proposition 2. Let $f:(0, \infty) \rightarrow \mathbb{R}$ be a convex function with the property that $f(1)=0$. Assume that $p, q \in \mathcal{P}$ and there exist constants $0<r<1<$ $R<\infty$ such that

$$
r \leq \frac{q(t)}{p(t)} \leq R \text { for } \mu \text {-a.e. } t \in \Omega .
$$

If $x \in[r, R]$, then we have the inequalities

$$
\begin{aligned}
\left|I_{f}(p, q)-f(x)\right| & \leq\left\|f^{\prime}\right\|_{[r, R], \infty} D_{v, x}(p, q) \\
& \leq\left\|f^{\prime}\right\|_{[r, R], \infty}\left[D_{\chi^{2}}(p, q)+(x-1)^{2}\right]^{1 / 2},
\end{aligned}
$$

where

$$
D_{v, x}(p, q):=\int_{\Omega}|p(t)-x q(t)| d \mu(t), \quad p, q \in \mathcal{P} .
$$

In particular, we have

$$
\begin{aligned}
\mid I_{f}(p, q) & -f\left(\frac{r+R}{2}\right) \mid \leq\left\|f^{\prime}\right\|_{[r, R], \infty} D_{v, \frac{r+R}{2}}(p, q) \\
& \leq\left\|f^{\prime}\right\|_{[r, R], \infty}\left[D_{\chi^{2}}(p, q)+\left(\frac{r+R}{2}-1\right)^{2}\right]^{1 / 2}
\end{aligned}
$$

and

$$
0 \leq I_{f}(p, q) \leq\left\|f^{\prime}\right\|_{[r, R], \infty} D_{v}(p, q) \leq\left\|f^{\prime}\right\|_{[r, R], \infty}\left[D_{\chi^{2}}(p, q)\right]^{1 / 2}
$$

Proof. Utilising the inequality (3.16) for the convex function $f$, we have

$$
\begin{aligned}
& \left|\int_{\Omega} p(t) f\left[\frac{q(t)}{p(t)}\right] d \mu(t)-f(x)\right| \\
& \leq\left\|f^{\prime}\right\|_{[r, R], \infty} \int_{\Omega}\left|\frac{q(t)}{p(t)}-x\right| p(t) d \mu(t) \\
& \leq\left\|f^{\prime}\right\|_{[r, R], \infty}\left[\int_{\Omega}\left(\frac{q(t)}{p(t)}\right)^{2} p(t) d \mu(t)-\left(\int_{\Omega} \frac{q(t)}{p(t)} p(t) d \mu(t)\right)^{2}\right. \\
& \left.\quad+\left(\int_{\Omega} \frac{q(t)}{p(t)} p(t) d \mu(t)-x\right)^{2}\right]^{1 / 2}
\end{aligned}
$$

which is equivalent to (5.12). 
We also have

Proposition 3. With the assumptions of Proposition 2 we have

$$
\begin{aligned}
& \left|I_{f}(p, q)-f(x)-\frac{f_{+}^{\prime}(r)+f_{-}^{\prime}(R)}{2}(1-x)\right| \\
& \quad \leq \frac{1}{2}\left[f_{-}^{\prime}(R)-f_{+}^{\prime}(r)\right] D_{v, x}(p, q)
\end{aligned}
$$

for any $x \in[r, R]$.

In particular, we have

$$
\begin{aligned}
& \left|I_{f}(p, q)-f\left(\frac{r+R}{2}\right)-\frac{f_{+}^{\prime}(r)+f_{-}^{\prime}(R)}{2}\left(1-\frac{r+R}{2}\right)\right| \\
& \quad \leq \frac{1}{2}\left[f_{-}^{\prime}(R)-f_{+}^{\prime}(r)\right] D_{v, \frac{r+R}{2}}(p, q)
\end{aligned}
$$

and

$$
0 \leq I_{f}(p, q) \leq \frac{1}{2}\left[f_{-}^{\prime}(R)-f_{+}^{\prime}(r)\right] D_{v}(p, q) .
$$

Proof. Utilising the inequality (4.12), we have

$$
\begin{aligned}
& \mid \int_{\Omega} p(t) f\left[\frac{q(t)}{p(t)}\right] d \mu(t)-f(x) \\
& \quad-\frac{f_{+}^{\prime}(r)+f_{-}^{\prime}(R)}{2}\left(\int_{\Omega} \frac{q(t)}{p(t)} p(t) d \mu(t)-x\right) \mid \\
& \quad \leq \frac{1}{2}\left[f_{-}^{\prime}(R)-f_{+}^{\prime}(r)\right] \int_{\Omega}\left|\frac{q(t)}{p(t)}-x\right| p(t) d \mu(t)
\end{aligned}
$$

for any $x \in[a, b]$, which is equivalent to (5.15).

If we consider the convex function $f:(0, \infty) \rightarrow \mathbb{R}, f(t)=t \ln t$, then

$$
\begin{aligned}
I_{f}(p, q) & :=\int_{\Omega} p(t) \frac{q(t)}{p(t)} \ln \left[\frac{q(t)}{p(t)}\right] d \mu(t)=\int_{\Omega} q(t) \ln \left[\frac{q(t)}{p(t)}\right] d \mu(t) \\
& =D_{K L}(q, p) .
\end{aligned}
$$

We have $f^{\prime}(t)=\ln t+1$ and by (5.17) we get

$$
0 \leq D_{K L}(q, p) \leq \ln \sqrt{\frac{R}{r}} D_{v}(p, q),
$$

provided that

$$
r \leq \frac{q(t)}{p(t)} \leq R \text { for } \mu \text {-a.e. } t \in \Omega
$$

If we consider the convex function $f:(0, \infty) \rightarrow \mathbb{R}, f(t)=-\ln t$, then

$$
\begin{aligned}
I_{f}(p, q) & :=-\int_{\Omega} p(t) \ln \left[\frac{q(t)}{p(t)}\right] d \mu(t)=\int_{\Omega} p(t) \ln \left[\frac{p(t)}{q(t)}\right] d \mu(t) \\
& =D_{K L}(p, q) .
\end{aligned}
$$


We have $f^{\prime}(t)=-\frac{1}{t}$ and by (5.17) we get

$$
0 \leq D_{K L}(p, q) \leq \frac{1}{2} \frac{R-r}{r R} D_{v}(p, q)
$$

provided that

$$
r \leq \frac{q(t)}{p(t)} \leq R \text { for } \mu \text {-a.e. } t \in \Omega
$$

\section{REFERENCES}

[1] Bhattacharyya, A., On a measure of divergence between two statistical populations defined by their probability distributions, Bull. Calcutta Math. Soc. 35 (1943), 99-109.

[2] Cerone, P., Dragomir, S. S., Midpoint-type rules from an inequalities point of view, in Handbook of Analytic-Computational Methods in Applied Mathematics, Anastassiou, G. A., (Ed.), CRC Press, New York, 2000, 135-200.

[3] Cerone, P., Dragomir, S. S., Roumeliotis, J., Some Ostrowski type inequalities for n-time differentiable mappings and applications, Demonstratio Math. 32 (2) (1999), 697-712.

[4] Csiszár, I. I., Information-type measures of difference of probability distributions and indirect observations, Studia Math. Hungarica 2 (1967), 299-318.

[5] Dragomir, S. S., Ostrowski's inequality for monotonous mappings and applications, J. KSIAM 3 (1) (1999), 127-135.

[6] Dragomir, S. S., The Ostrowski's integral inequality for Lipschitzian mappings and applications, Comp. Math. Appl. 38 (1999), 33-37.

[7] Dragomir, S. S., The Ostrowski integral inequality for mappings of bounded variation, Bull. Austral. Math. Soc. 60 (1) (1999), 495-508.

[8] Dragomir, S. S., A converse result for Jensen's discrete inequality via Gruss' inequality and applications in information theory, An. Univ. Oradea Fasc. Mat. 7 (1999/2000), 178-189.

[9] Dragomir, S. S., On the Ostrowski's integral inequality for mappings with bounded variation and applications, Math. Inequal. Appl. 4 (1) (2001), 59-66.

[10] Dragomir, S. S., On a reverse of Jessen's inequality for isotonic linear functionals, J. Ineq. Pure Appl. Math. 2, No. 3, (2001), Art. 36.

[11] Dragomir, S. S., An Ostrowski like inequality for convex functions and applications, Revista Math. Complutense 16 (2) (2003), 373-382.

[12] Dragomir, S. S., Reverses of the Jensen inequality in terms of the first derivative and applications, Acta Math. Vietnam. 38, no. 3 (2013), 429-446. Preprint RGMIA Res. Rep. Coll. 14 (2011), Art. 71. [Online http://rgmia.org/papers/v14/v14a71.pdf].

[13] Dragomir, S. S., Operator Inequalities of Ostrowski and Trapezoidal Type, Springer, New York, 2012.

[14] Dragomir, S. S., Perturbed companions of Ostrowski's inequality for absolutely continuous functions (I), An. Univ. Vest Timiş. Ser. Mat.-Inform. 54, no. 1 (2016), 119-138. Preprint RGMIA Res. Rep. Coll. 17 (2014), Art 7, 15 pp. [Online http://rgmia.org/papers/v17/v17a07.pdf].

[15] Dragomir, S. S., General Lebesgue integral inequalities of Jensen and Ostrowski type for differentiable functions whose derivatives in absolute value are $h$-convex and applications, Ann. Univ. Mariae Curie-Skłodowska Sect. A 69, no. 2 (2015), 17-45.

[16] Dragomir, S. S., Cerone, P., Roumeliotis, J., Wang, S., A weighted version of Ostrowski inequality for mappings of Hölder type and applications in numerical analysis, Bull. Math. Soc. Sci. Math. Romanie 42(90) (4) (1999), 301-314. 
[17] Dragomir, S. S., Ionescu, N. M., Some converse of Jensen's inequality and applications, Rev. Anal. Numér. Théor. Approx. 23, No. 1 (1994), 71-78.

[18] Dragomir, S. S., Rassias, Th. M. (Eds.), Ostrowski Type Inequalities and Applications in Numerical Integration, Kluwer Academic Publishers, Dordrecht-Boston-London, 2002.

[19] Hellinger, E., Neue Bergrüirdung du Theorie quadratisher Formerus von uneudlichvieleu Veränderlicher, J. Reine Angew. Math. 36 (1909), 210-271.

[20] Jeffreys, H., An invariant form for the prior probability in estimating problems, Proc. Roy. Soc. London A Math. Phys. Sci. 186 (1946), 453-461.

[21] Kapur, J. N., A comparative assessment of various measures of directed divergence, Advances in Management Studies 3 (1984), 1-16.

[22] Kullback, S., Leibler, R. A., On information and sufficiency, Annals Math. Statist. 22 (1951), 79-86.

[23] Ostrowski, A., Über die Absolutabweichung einer differentienbaren Funktionen von ihren Integralmittelwert, Comment. Math. Helv. 10 (1938), 226-227.

[24] Taneja, I. J., Generalised Information Measures and Their Applications [Online http://www.mtm.ufsc.br/ taneja/bhtml/bhtml.html].

[25] Topsoe, F., Some inequalities for information divergence and related measures of discrimination, Preprint RGMIA Res. Rep. Coll. 2 (1) (1999), 85-98.

S. S. Dragomir

Mathematics, College of Engineering \& Science

Victoria University, PO Box 14428

Melbourne City, MC 8001

Australia

DST-NRF Centre of Excellence in the Mathematical and Statistical Sciences

School of Computational \& Applied Mathematics

University of the Witwatersrand

Private Bag 3, Johannesburg 2050

South Africa

e-mail: sever.dragomir@vu.edu.au

urladdr: http://rgmia.org/dragomir

Received March 22, 2016 